\title{
Validation of the unruptured intracranial aneurysm treatment score: comparison with real-world cerebrovascular practice
}

\author{
Vijay M. Ravindra, MD, MSPH, ${ }^{1}$ Adam de Havenon, MD, ${ }^{2}$ Timothy C. Gooldy, MD, ${ }^{3}$ \\ Jonathan Scoville, MD, ${ }^{1}$ Jian Guan, MD, ${ }^{1}$ William T. Couldwell, MD, PhD, ${ }^{1}$ Philipp Taussky, MD, ${ }^{1}$ \\ Joel D. MacDonald, MD, ${ }^{1}$ Richard H. Schmidt, MD, PhD, ${ }^{1}$ and Min S. Park, MD1 \\ Departments of ${ }^{1}$ Neurosurgery and ${ }^{2}$ Neurology, Clinical Neurosciences Center, University of Utah, Salt Lake City, Utah; and \\ ${ }^{3}$ Department of Neurosurgery, University of Florida School of Medicine, Gainesville, Florida
}

\begin{abstract}
OBJECTIVE The purpose of this study was to compare the unruptured intracranial aneurysm treatment score (UIATS) recommendations with the real-world experience in a quaternary academic medical center with a high volume of patients with unruptured intracranial aneurysms (UIAs).

METHODS All patients with UIAs evaluated during a 3-year period were included. All factors included in the UIATS were abstracted, and patients were scored using the UIATS. Patients were categorized in a contingency table assessing UIATS recommendation versus real-world treatment decision. The authors calculated the percentage of misclassification, sensitivity, specificity, and area under the receiver operating characteristic $(\mathrm{ROC})$ curve.

RESULTS A total of 221 consecutive patients with UIAs met the inclusion criteria: 69 (31\%) patients underwent treatment and $152(69 \%)$ did not. Fifty-nine (27\%) patients had a UIATS between -2 and 2, which does not offer a treatment recommendation, leaving $162(73 \%)$ patients with a UIATS treatment recommendation. The UIATS was significantly associated with treatment $(p<0.001)$; however, the sensitivity, specificity, and percentage of misclassification were $49 \%$, $80 \%$, and $28 \%$, respectively. Notably, $51 \%$ of patients for whom treatment would be recommended by the UIATS did not undergo treatment in the real-world cohort and $20 \%$ of patients for whom conservative management would be recommended by UIATS had intervention. The area under the ROC curve was 0.646 .
\end{abstract}

CONCLUSIONS Compared with the authors' experience, the UIATS recommended overtreatment of UIAs. Although the UIATS could be used as a screening tool, individualized treatment recommendations based on consultation with a cerebrovascular specialist are necessary. Further validation with longitudinal data on rupture rates of UIAs is needed before widespread use.

https://thejns.org/doi/abs/10.3171/2017.4.JNS17548

KEY WORDS stroke; unruptured intracranial aneurysm treatment score; UIATS; vascular disorders

$\mathrm{U}$ NRUPTURED intracranial aneurysms (UIAs) are seen in nearly $3 \%$ of the adult population, with a rising prevalence secondary to increased utilization of intracranial vascular imaging., ${ }^{9,25}$ Although thorough natural history studies are lacking, there is evidence that a small proportion of UIAs rupture, causing subarachnoid hemorrhage (SAH). ${ }^{6,13,14,18,19,21,26,27}$ Although previous reports are biased by aneurysm location, it has been found that small UIAs especially have a low risk of rupture. ${ }^{6}$ Because of the higher prevalence of small aneurysms, a majority of aneurysmal cases of SAH are due to small aneurysms. Unfortunately, the specific factors associated with those small UIAs that proceed to rupture are not known.

The management of UIAs is subject to provider bias supported by data with varying levels of evidence; thus,

ABBREVIATIONS AUC $=$ area under the curve; $\mathrm{CC}=$ correlation coefficient; $\mathrm{CCI}=$ Charlson Comorbidity Index; $\mathrm{ROC}=$ receiver operating characteristic; $\mathrm{SAH}=$ subarachnoid hemorrhage; UIA = unruptured intracranial aneurysm; UIATS = UIA treatment score.

SUBMITTED March 2, 2017. ACCEPTED April 14, 2017.

INCLUDE WHEN CITING Published online October 6, 2017; DOI: 10.3171/2017.4.JNS17548. 
there is substantial variation in the management of UIAs among clinicians. ${ }^{3,5}$ The unruptured intracranial aneurysm treatment score (UIATS) was developed by 69 multidisciplinary specialists to guide clinical decision making in patients with UIAs. ${ }^{6}$ The score represents a consensus opinion regarding aneurysm management, rather than a scientific analysis of whether the aneurysm should be treated. The purpose of this investigation was to compare the UIATS with the real-world experience of a quaternary academic medical center with a high volume of patients with UIAs. We hypothesized that there would be a discrepancy when comparing the UIATS recommendation with the real-world experience of a quaternary academic high-volume center.

\section{Methods}

\section{Patient Cohort}

After obtaining IRB approval, we retrospectively queried a prospective database of all patients older than 18 years harboring a diagnosed UIA evaluated at the University of Utah Clinical Neurosciences Center from January 1, 2013, through December 31, 2015. A chart review was performed to abstract all 29 factors in the UIATS (Fig. 1). We also collected information about whether patients received treatment or were managed with observation. $\mathrm{Pa}-$ tients who were missing any of the 29 variables from the UIATS were excluded from analysis. All treatment recommendations were made, and subsequent treatments performed, by one of 5 senior attending neurosurgeons with experience in treating complex cerebrovascular disorders, with a practice focused on cerebral aneurysms (W.T.C., R.H.S., J.D.M., P.T., and M.S.P.). The decision-making process is multifactorial, but it is not standardized. Patient involvement is crucial to the decision-making process, but no uniform set of factors is used among surgeons.

\section{Data Collection and UIATS Variables}

Each of the 29 variables in the UIATS was abstracted from the patients' medical records. Demographic information included age (dichotomous by age $<65$ years vs $\geq 65$ years), sex, and race. Clinical information included whether the patient had experienced a previous SAH or had a history of hypertension, coagulopathies, epilepsy, and neurocognitive disorders. Aneurysm-specific information included multiplicity, presence of multilobulation, size in millimeters, location, thromboembolic events due to the aneurysm, mass effect, size ratio, aspect ratio, new aneurysm formation on imaging, contralateral steno-occlusive disease, and associated cranial nerve deficits. The high-complexity versus low-complexity variable was calculated by a neurosurgeon who was blinded to the treatment decision after reviewing the patient's medical record and neuroimaging findings. Additional comorbidity data included active tobacco use, active alcohol use, and illicit drug use. Family history information about cerebral aneurysms, previous aneurysmal SAH, multiple intracranial aneurysms, and other disorders associated with intracranial aneurysm development and/or rupture (polycystic kidney disease, hereditary hemorrhagic telangiectasia, arteriovenous malformations) was also collected. A Charlson
Comorbidity Index (CCI) score was calculated for each patient. ${ }^{4}$ The CCI was used to estimate the life expectancy of patients, with scores of 0-3 treated as $>10$ years, those 4-8 as 5-10 years, and those $>8$ as $<5$ years. ${ }^{1,2,17}$

\section{Statistical Analysis}

All data analyses were carried out in a de-identified manner. Data were summarized using means and standard deviations for continuous variables and counts and frequencies for categorical variables. The demographic, clinical, and radiographic variables were compared among centers using the Student t-test for continuous variables and the chi-square test for categorical variables.

The validation analysis, performed by one person, was done by scoring each patient in the validation cohort using the UIATS. After scoring, patients were classified as UIATS recommends treatment (score $\geq 3$; i.e., "Favors UIA repair" score was more than 3 points greater than "Favors UIA conservative management" score) or UIATS did not recommend treatment (score $\leq-3$; i.e., "Favors UIA conservative management" score was more than 3 points greater than "Favors UIA repair" score), and no treatment recommendation (UIATS between -2 and 2; i.e., difference between "Favors UIA conservative management" score and "Favors UIA repair" score was $\leq 2$ in either direction). Patients whose scores fell between -2 and 2 were excluded because the UIATS did not provide a recommendation. The remaining patients were categorized in a contingency table assessing UIATS recommendation versus the real-world treatment decision. We calculated the percentage of misclassification, sensitivity and specificity, and area under the receiver operating characteristic (ROC) curve for the UIATS in comparison with real-world experience. This comparison was analyzed using the chi-square test. We calculated the percentage of misclassification, sensitivity, specificity, positive predictive value, and negative predictive value. Before the study, validation was defined as a misclassification rate of $<25 \%$. $^{20}$

A logistic regression model was developed for the UIATS risk categorization and its association with realworld treatment. Using this model, we calculated the area under the curve (AUC) for discrimination of the dichotomized UIATS. To determine which elements of the UIATS best predicted the real-world treatment decision, a correlation coefficient (CC) was calculated between each of the individual components of the UIATS and the realworld treatment decision for the entire cohort. Data were analyzed using Stata (version 12.1, StataCorp.).

\section{Results}

A total of 221 consecutive patients with UIAs met the inclusion criteria: 69 of $221(31 \%)$ patients were treated and 152 of $221(69 \%)$ were not treated. Fifty-nine patients (27\%) had a UIATS between -2 and 2, which does not offer a treatment recommendation, leaving 162 of $221(73 \%)$ patients who had a UIATS treatment recommendation for inclusion. The scores of 117 patients indicated UIATS recommendations of no treatment, whereas the scores of 45 would recommend treatment. The mean patient age of the 


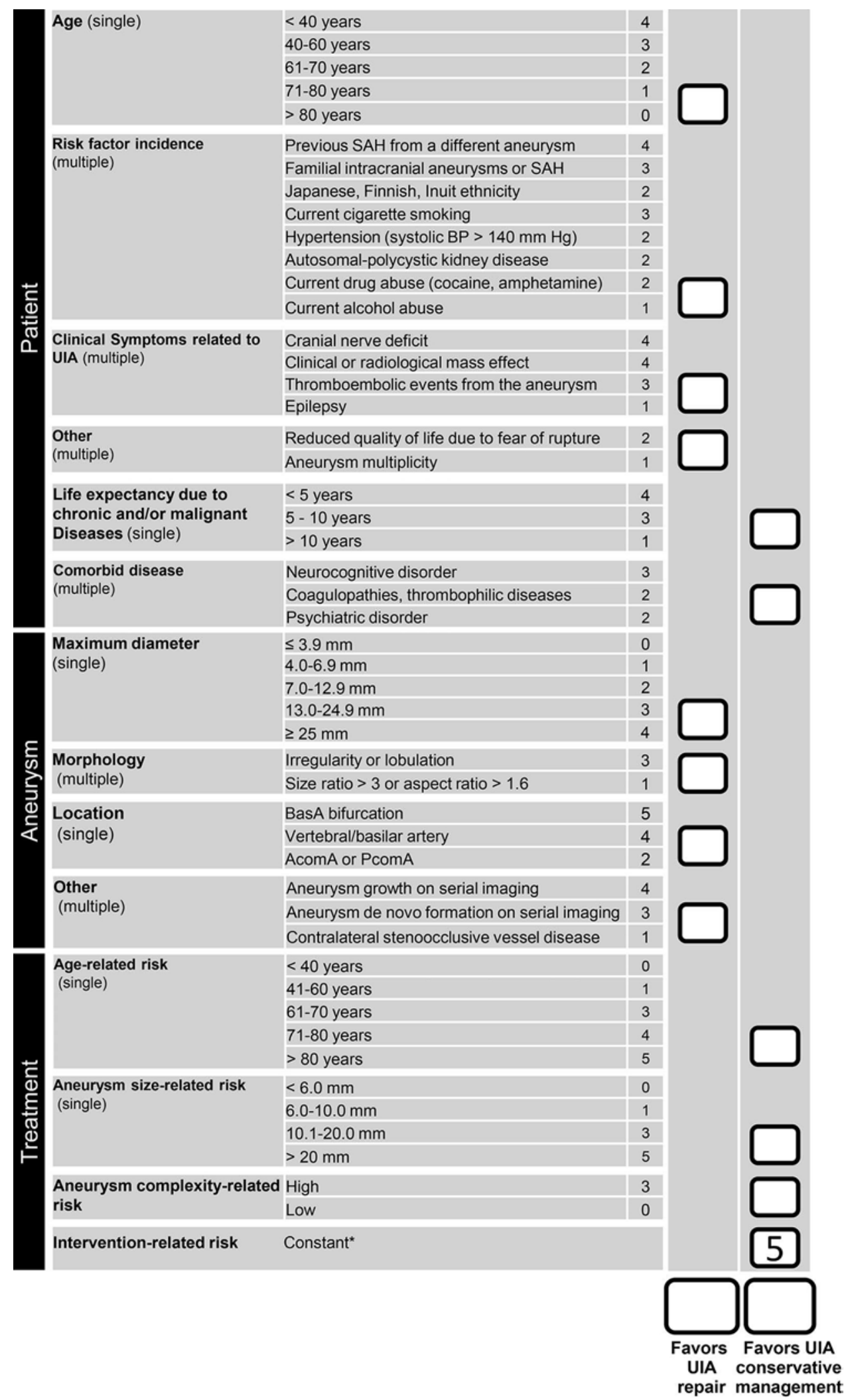

FIG. 1. The UIATS. To calculate a management recommendation for a UIA, the number of points corresponding to each patient-, aneurysm-, or treatment-related feature in both management columns of the scoring form ("In favor of UIA repair" and "In favor of UIA conservative management") are totaled. This will lead to 2 numerical values, one favoring aneurysm repair (surgical or endovascular) and the other favoring conservative management. Reproduced with permission from Etminan N, Brown RD Jr, Beseoglu $\mathrm{K}$, Juvela S, Raymond J, Morita A, et al: The unruptured intracranial aneurysm treatment score: a multidisciplinary consensus. Neurology 85(10):881-889, 2015. http://www.neurology.org/content/85/10/881.long. 
TABLE 1. Demographic information for UIATS recommending treatment versus no treatment

\begin{tabular}{|c|c|c|c|}
\hline \multirow[b]{2}{*}{ Variable } & \multicolumn{3}{|c|}{ UIATS Recommendation } \\
\hline & No Treatment & Treatment & No Recommendation \\
\hline No. of patients & 117 & 45 & 59 \\
\hline Mean age in yrs $( \pm S D)$ & $69.9 \pm 12.32$ & $50.8 \pm 14$ & $56.6 \pm 13.9$ \\
\hline Female & $81(69)$ & $34(76)$ & $43(73)$ \\
\hline \multicolumn{4}{|l|}{ Risk factor incidence } \\
\hline Previous SAH from different aneurysm & 0 & 0 & $1(1.7)$ \\
\hline Family history of aneurysmal SAH & $1(0.85)$ & $3(6.6)$ & $8(13.5)$ \\
\hline Japanese, Finnish, Inuit ethnicity & $1(0.85)$ & $1(2.2)$ & 0 \\
\hline Caucasian & $88(75)$ & $35(77)$ & $45(76)$ \\
\hline Current cigarette smoker & $3(2.5)$ & $19(42)$ & $7(12)$ \\
\hline Hypertension & $69(59)$ & $23(51)$ & $34(58)$ \\
\hline Alcohol use & $25(21)$ & $17(38)$ & $13(22)$ \\
\hline Illicit drug use & 0 & $6(13.3)$ & 0 \\
\hline \multicolumn{4}{|l|}{ Clinical symptoms related to UIA } \\
\hline Cranial nerve deficit & $3(2.5)$ & $9(20)$ & $3(5)$ \\
\hline Clinical or radiological mass effect & $6(5)$ & $8(18)$ & $7(12)$ \\
\hline Thromboembolic events from aneurysm & $22(19)$ & $12(27)$ & $16(27)$ \\
\hline Epilepsy & $8(6.8)$ & $2(4.4)$ & $6(10)$ \\
\hline \multicolumn{4}{|l|}{ Other } \\
\hline Reduced quality of life due to fear of rupture & $5(4.3)$ & $5(11)$ & $2(3.4)$ \\
\hline Aneurysm multiplicity & $10(8.5)$ & $13(29)$ & $12(20)$ \\
\hline \multicolumn{4}{|l|}{ Comorbid disease } \\
\hline Neurocognitive disorder & $15(12.8)$ & $2(4.4)$ & $1(1.7)$ \\
\hline Coagulopathies/thrombophilic disease & $5(4.2)$ & 0 & $5(8.4)$ \\
\hline \multicolumn{4}{|l|}{ Morphology } \\
\hline Mean aneurysm diameter in $\mathrm{mm}( \pm \mathrm{SD})$ & $5.4 \pm 3.9$ & $5.4 \pm 2.62$ & $7.05 \pm 7.48$ \\
\hline Multilobulated aneurysm & $14(12)$ & $20(44)$ & $16(27)$ \\
\hline Size ratio $>3$ or aspect ratio $>1.6$ & $16(14)$ & $8(18)$ & $12(20)$ \\
\hline Aneurysm $<7 \mathrm{~mm}$ & $27(23)$ & $14(31)$ & $22(37)$ \\
\hline \multicolumn{4}{|l|}{ Location } \\
\hline Basilar bifurcation or vertebral/basilar artery & $1(0.85)$ & $8(18)$ & $2(3.3)$ \\
\hline Anterior or posterior communicating artery & $27(23)$ & $16(36)$ & $16(27)$ \\
\hline \multicolumn{4}{|l|}{ Other } \\
\hline Aneurysm growth on serial imaging & $5(4.3)$ & 0 & $4(6.8)$ \\
\hline De novo formation on serial imaging & 0 & $8(18)$ & $2(3.4)$ \\
\hline Contralateral steno-occlusive disease & 0 & $1(2.2)$ & $2(3.4)$ \\
\hline High aneurysm complexity & $1(1)$ & $2(4.4)$ & $3(5.1)$ \\
\hline No. of UIAs treated in our real-world scenario & $23(19.6)$ & $22(49)$ & $24(41)$ \\
\hline
\end{tabular}

Values are presented as the number of patients (\%) unless indicated otherwise.

group for which treatment was not recommended was 70 years, and that of the group for which treatment was recommended was 51 years. The majority of patients in both groups were female (Table 1).

The UIATS recommendation was significantly associated with the actual treatment of UIAs ( $<0.001)$; the sensitivity of the UIATS was $49 \%$, specificity was $80 \%$, and percentage of misclassification was $28 \%$ (Table 2). Notably, $51 \%$ of patients in whom the UIATS would recommend treatment were untreated in our cohort and $20 \%$ of patients for whom the UIATS would recommend conservative management had intervention. No patient in the conservatively managed group experienced a subsequent SAH due to aneurysm rupture during the follow-up period of up to 4 years. The AUC was suboptimal at 0.646 (Fig. 2).

The correlation between the individual elements of the UIATS and real-world decision to recommend treatment was significant for older age $(\mathrm{CC}=0.161, \mathrm{p}=0.016)$, patient fear of rupture $(\mathrm{CC}=0.170, \mathrm{p}=0.012)$, multilobulated aneurysm $(\mathrm{CC}=0.216, \mathrm{p}<0.001)$, larger aneurysm size 
TABLE 2. Contingency table of UIATS compared with real-world treatment decisions

\begin{tabular}{lccc}
\hline & UIATS $\leq-3^{*}$ & UIATS $\geq 3 \dagger$ & UIATS -2 to $2 \ddagger$ \\
\hline Untreated UIA & $94(80)$ & $23(51)$ & $35(59)$ \\
\hline Treated UIA & $23(20)$ & $22(49)$ & $24(41)$ \\
\hline
\end{tabular}

Values are presented as the number of patients (\%). The sensitivity of the UIATS was $49 \%$, specificity was $80 \%$, and percent misclassification was $28 \%$. The area under the ROC curve was suboptimal at 0.646 .

* UIATS recommendation of no treatment.

$\dagger$ UIATS recommendation of treatment.

$\ddagger$ No UIATS recommendation.

$(\mathrm{CC}=0.196, \mathrm{p}=0.003)$, and size ratio $>3$ or aspect ratio $>$ $1.6(\mathrm{CC}=0.258, \mathrm{p}<0.001)$.

\section{Discussion}

The management of UIAs is a controversial topic in the cerebrovascular literature. There is a large body of work with conflicting recommendations, and careful review of the literature regarding risk factors and modeling based on patient characteristics can be difficult in part because of the varying degrees of evidence. ${ }^{11}$ A considerable effort has been put forth to guide clinical management and decision making by the creation of the UIATS, ${ }^{6}$ but this scoring system has not been broadly adopted. Our effort to compare this instrument with the experience of a single, high-volume, quaternary, academic medical center demonstrates several differences.

The UIATS was proposed by a multidisciplinary consensus group to aid management and decision making for patients with UIAs. ${ }^{6}$ On its conception and in the initial report, the authors offered internal and external groups of patients for validation using a Delphi consensus. They also selected 30 UIA cases to analyze agreement with the UIATS based on a 5-point Likert scale. The authors found a high level of agreement of the 30 cases with the UIATS and high interrater agreement among the specialist group that devised the score and thus concluded that the UIATS captured excellent consensus of UIA management and endorsed its use for managing individual patients with UIAs. ${ }^{6}$

The UIATS differs from the PHASES score, which is a model based on prospectively collected data from 6 cohort studies on risk of UIA rupture that provides risks of rupture for the initial 5 years after aneurysm detection using 6 easily retrievable baseline characteristics (patient geographic location and age, aneurysm size and location, hypertension, and previous SAH from a different aneurysm)..$^{10}$ A criticism of the PHASES score, which has limited its use and generalizability, was that some groups of patients, specifically those with familial aneurysms or young smokers, were underrepresented; ${ }^{6}$ an additional criticism comes from the lack of long-term follow-up $(>5$ years) and the exclusion of the risk of intervention or treatment, which the UIATS includes. Thus, the UIATS was designed to address these shortcomings and "harmonize" the variability seen among clinicians managing UIAs.

The factors used in the UIATS include many that have not been studied in previous observational or cohort studies (young age or long life expectancy, coexisting modifiable and unmodifiable risk factors, comorbidities, aneurysm characteristics, and clinical symptoms related to the UIA). ${ }^{715,16,18,22,24}$ The instrument was created using the Delphi method to reach consensus, which is accepted as a

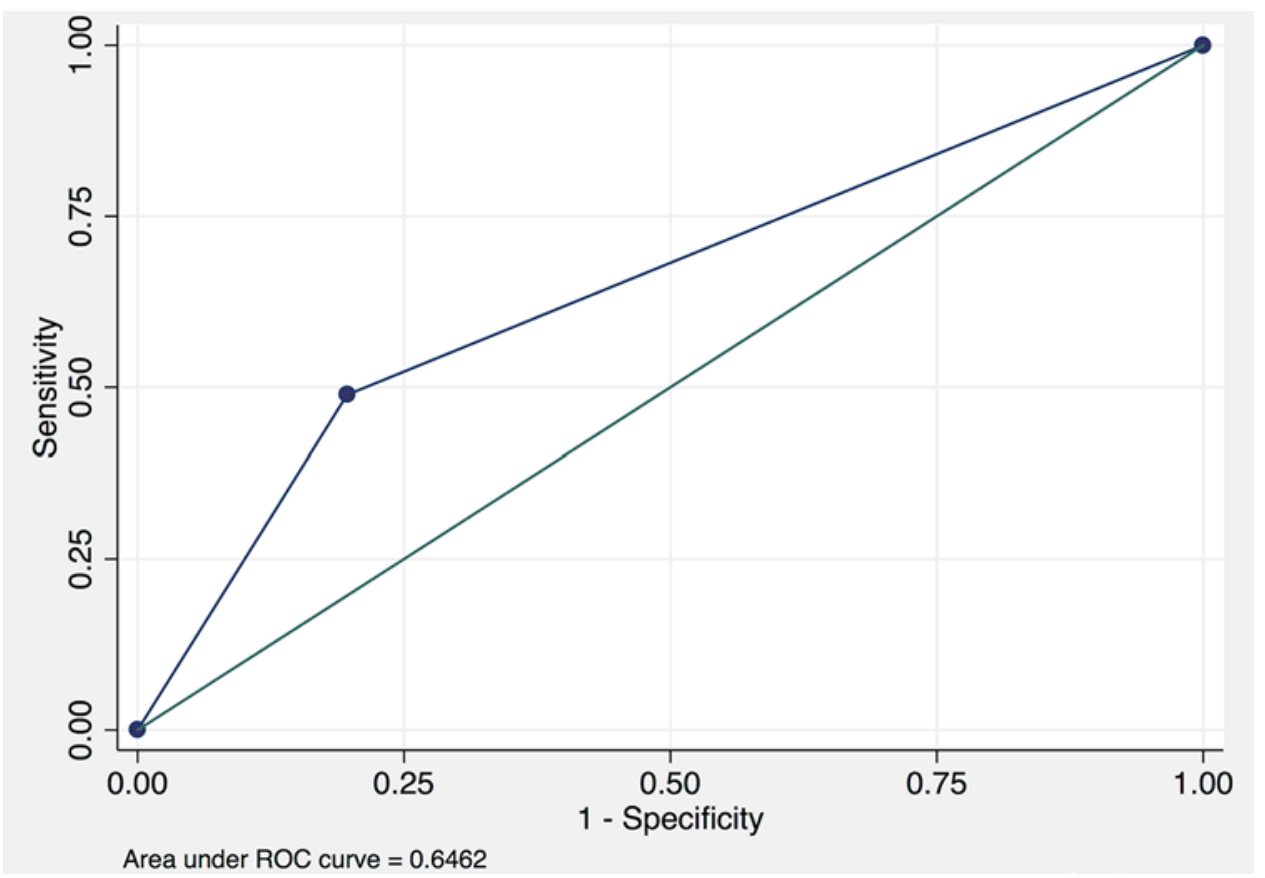

FIG. 2. The ROC demonstrating an AUC of 0.646 , indicating a low level of accuracy of the UIATS in comparison with real-world treatment decisions. Figure is available in color online only. 
scientifically sound methodology and has been used to develop treatment scores on similarly complex subjects..$^{8,12,28}$ The authors of the UIATS suggest the use of the UIATS as a complement to evidence, but our results indicate that there are discrepancies between the UIATS and the reality of a large cerebrovascular practice. There may be additional factors or persuading information that is left out of the UIATS. Ultimately, consultation with a specialist is necessary for any UIA.

Using a prospectively populated database, we found a low sensitivity of $49 \%$, specificity of $80 \%$, and percentage of misclassification of $28 \%$ when we compared the UIATS with the real-world decision of whether to treat a UIA. Low sensitivity is not desirable for a screening tool of this nature. In fact, if the UIATS had been used to make treatment decisions, rather than further diagnostic decisions in this cohort of patients, this low sensitivity would result in a poor performance when matched against the experience of 5 cerebrovascular neurosurgeons and would result in overtreatment in comparison. Nearly $51 \%$ of patients in our cohort for whom treatment would be recommended by the UIATS were untreated, thus suggesting that the UIATS would have recommended overtreatment in our cohort. Notably, no patient in the cohort of conservatively managed patients had a subsequent $\mathrm{SAH}$ from aneurysm rupture during a follow-up period of up to 4 years. This finding and interpretation should be taken carefully, however, given that this is the reflection of a single academic center. In addition, $20 \%$ of patients in our cohort for whom conservative management would be recommended by UIATS underwent intervention. This 2-sided mismatch indicates that there may be additional factors that influence decisions about treatment that are not reflected in the UIATS. Moreover, the overall performance of the model, despite being statistically significant, was quite low, with an AUC of 0.646, indicating discordance in the comparison between the UIATS and our experience.

Using multivariable regression analysis of a larger cohort of 424 patients that included the 221 patients described in this report, Guan et al. ${ }^{11}$ found that the presence of multiple aneurysms (OR 2.093), larger aneurysm size (OR 1.176), lower CCI (OR 1.536), and younger age (OR 2.913) were associated with the decision to pursue treatment. All of these factors are included, to some degree, in the UIATS, but the performance of the UIATS score on this testing cohort was suboptimal.

For this cohort, we calculated a CC between the individual elements of the UIATS and the real-world decision to recommend treatment. We found that there was significant correlation for older age, patient fear of rupture, multilobulated aneurysm, larger aneurysm size, and size ratio $>3$ or aspect ratio $>1$.6. This indicates that these variables may play a large role in the practitioner's decision to treat an aneurysm, and could be included, and weighted more heavily, in a potential treatment algorithm or scheme.

We theorize that the discrepancy between the UIATS recommendation and the actual treatment that patients received is likely multifactorial. The correlation between treatment and UIATS yielded older age, greater patient fear of rupture, multilobulated aneurysm, larger aneurysm size, and size ratio $>3$ or aspect ratio $>1.6$ as factors that were significant based on our single-center cohort of patients. It could be that the practice pattern at our center differs from those involved in the creation of the UIATS, but it is unlikely that this alone accounts for the discrepancy. Factors that are considered when evaluating UIAs include size, irregularity of the aneurysm (geometry), family history, growth over time, and patient anxiety, as well as other comorbidities. Although the UIATS attempts to address anxiety and patient fear, the "human factor" clearly plays a role and is based on patient anxiety surrounding the lesion and its effect on quality of life, ${ }^{23}$ as well as the interaction with the surgeon in the context of current treatment recommendations. This set of factors is difficult to quantify/ qualify, but at our center it plays a large role in deciding whether to treat or observe over time. Because of the degree of discrepancy, the suggestion based on the current study is that consultation with a cerebrovascular specialist is necessary in all cases of UIAs.

\section{Limitations}

This single-center validation study was conducted on a retrospective cohort from a prospectively collected database, which may limit the accuracy and availability of the medical record. This study demonstrated a poor comparison between the UIATS and real-world treatment decisions, but the data presented represent only a singlecenter practice with 5 experienced cerebrovascular neurosurgeons. As with any evaluation of treatment decisions, patient and physician interpretations of the available information play a large role and may be subject to bias. Thus, treatment decisions may be biased by individual providers since no uniform treatment algorithm is used; however, the UIATS is based on consensus opinion, not a scientific analysis and thus is also a subjective measure. Further validation or study of the UIATS at multiple sites may be beneficial. Ultimately, prospective tracking and use of the UIATS is necessary prior to widespread use and implementation. We advise that clinical evaluation and multidisciplinary decision making should be paramount when making all diagnostic and treatment decisions.

\section{Conclusions}

This single-center comparison of the UIATS with a real-world experience found that UIATS generally recommended overtreatment of UIAs. Although the UIATS could be used as a screening tool, a further prospective validation of the UIATS is needed prior to widespread use.

\section{Acknowledgments}

We thank Kristin Kraus, MSc, for editorial assistance.

\section{References}

1. Bradshaw PJ, Stobie P, Knuiman MW, Briffa TG, Hobbs MS: Life expectancy after implantation of a first cardiac permanent pacemaker (1995-2008): A population-based study. Int J Cardiol 190:42-46, 2015

2. Brilleman SL, Salisbury C: Comparing measures of multimorbidity to predict outcomes in primary care: a cross sectional study. Fam Pract 30:172-178, 2013 
3. Brown RD Jr, Broderick JP: Unruptured intracranial aneurysms: epidemiology, natural history, management options, and familial screening. Lancet Neurol 13:393-404, 2014

4. Charlson ME, Pompei P, Ales KL, MacKenzie CR: A new method of classifying prognostic comorbidity in longitudinal studies: development and validation. J Chronic Dis 40:373383, 1987

5. Etminan N, Beseoglu K, Barrow DL, Bederson J, Brown RD $\mathrm{Jr}$, Connolly ES Jr, et al: Multidisciplinary consensus on assessment of unruptured intracranial aneurysms: proposal of an international research group. Stroke 45:1523-1530, 2014

6. Etminan N, Brown RD Jr, Beseoglu K, Juvela S, Raymond $\mathrm{J}$, Morita A, et al: The unruptured intracranial aneurysm treatment score: a multidisciplinary consensus. Neurology 85:881-889, 2015

7. Etminan N, Dreier R, Buchholz BA, Beseoglu K, Bruckner P, Matzenauer C, et al: Age of collagen in intracranial saccular aneurysms. Stroke 45:1757-1763, 2014

8. Fisher CG, DiPaola CP, Ryken TC, Bilsky MH, Shaffrey CI, Berven SH, et al: A novel classification system for spinal instability in neoplastic disease: an evidence-based approach and expert consensus from the Spine Oncology Study Group. Spine (Phila Pa 1976) 35:E1221-E1229, 2010

9. Gabriel RA, Kim H, Sidney S, McCulloch CE, Singh V, Johnston SC, et al: Ten-year detection rate of brain arteriovenous malformations in a large, multiethnic, defined population. Stroke 41:21-26, 2010

10. Greving JP, Wermer MJ, Brown RD Jr, Morita A, Juvela S, Yonekura M, et al: Development of the PHASES score for prediction of risk of rupture of intracranial aneurysms: a pooled analysis of six prospective cohort studies. Lancet Neurol 13:59-66, 2014

11. Guan J, Karsy M, Couldwell WT, Schmidt RH, Taussky P, MacDonald JD, et al: Factors influencing management of unruptured intracranial aneurysms: an analysis of 424 consecutive patients. J Neurosurg 127:96-101, 2017

12. Hasson F, Keeney S, McKenna H: Research guidelines for the Delphi survey technique. J Adv Nurs 32:1008-1015, 2000

13. Ishibashi T, Murayama Y, Urashima M, Saguchi T, Ebara M, Arakawa $\mathrm{H}$, et al: Unruptured intracranial aneurysms: incidence of rupture and risk factors. Stroke 40:313-316, 2009

14. Juvela S, Poussa K, Lehto H, Porras M: Natural history of unruptured intracranial aneurysms: a long-term follow-up study. Stroke 44:2414-2421, 2013

15. Koffijberg H, Buskens E, Algra A, Wermer MJ, Rinkel GJ: Growth rates of intracranial aneurysms: exploring constancy. J Neurosurg 109:176-185, 2008

16. Korja M, Lehto H, Juvela S: Lifelong rupture risk of intracranial aneurysms depends on risk factors: a prospective Finnish cohort study. Stroke 45:1958-1963, 2014

17. Md Yusof MY, Horan MA, Jones M, McInnes L, Rabbitt PM, Pendleton N: Developing a self-reported comorbidity index to predict mortality of community-dwelling older adults. Arch Gerontol Geriatr 50:e63-e67, 2010

18. Morita A, Kirino T, Hashi K, Aoki N, Fukuhara S, Hashimoto N, et al: The natural course of unruptured cerebral aneurysms in a Japanese cohort. N Engl J Med 366:2474-2482, 2012

19. Nieuwkamp DJ, Setz LE, Algra A, Linn FH, de Rooij NK,
Rinkel GJ: Changes in case fatality of aneurysmal subarachnoid haemorrhage over time, according to age, sex, and region: a meta-analysis. Lancet Neurol 8:635-642, 2009

20. Riva-Cambrin J, Detsky AS, Lamberti-Pasculli M, Sargent MA, Armstrong D, Moineddin R, et al: Predicting postresection hydrocephalus in pediatric patients with posterior fossa tumors. J Neurosurg Pediatr 3:378-385, 2009

21. Sonobe M, Yamazaki T, Yonekura M, Kikuchi H: Small unruptured intracranial aneurysm verification study: SUAVe study, Japan. Stroke 41:1969-1977, 2010

22. Ujiie H, Tachibana H, Hiramatsu O, Hazel AL, Matsumoto T, Ogasawara Y, et al: Effects of size and shape (aspect ratio) on the hemodynamics of saccular aneurysms: a possible index for surgical treatment of intracranial aneurysms. Neurosurgery 45:119-130, 1999

23. van der Schaaf IC, Brilstra EH, Rinkel GJ, Bossuyt PM, van Gijn J: Quality of life, anxiety, and depression in patients with an untreated intracranial aneurysm or arteriovenous malformation. Stroke 33:440-443, 2002

24. Villablanca JP, Duckwiler GR, Jahan R, Tateshima S, Martin NA, Frazee J, et al: Natural history of asymptomatic unruptured cerebral aneurysms evaluated at CT angiography: growth and rupture incidence and correlation with epidemiologic risk factors. Radiology 269:258-265, 2013

25. Vlak MH, Algra A, Brandenburg R, Rinkel GJ: Prevalence of unruptured intracranial aneurysms, with emphasis on sex, age, comorbidity, country, and time period: a systematic review and meta-analysis. Lancet Neurol 10:626-636, 2011

26. Wermer MJ, van der Schaaf IC, Velthuis BK, Majoie CB, Albrecht KW, Rinkel GJ: Yield of short-term follow-up CT/ MR angiography for small aneurysms detected at screening. Stroke 37:414-418, 2006

27. Wiebers DO, Whisnant JP, Huston J III, Meissner I, Brown RD Jr, Piepgras DG, et al: Unruptured intracranial aneurysms: natural history, clinical outcome, and risks of surgical and endovascular treatment. Lancet 362:103-110, 2003

28. Zafar SY, Currow DC, Cherny N, Strasser F, Fowler R, Abernethy AP: Consensus-based standards for best supportive care in clinical trials in advanced cancer. Lancet Oncol 13:e77-e82, 2012

\section{Disclosures}

Dr. Taussky reports that he is a consultant for Medtronic.

\section{Author Contributions}

Conception and design: Park. Acquisition of data: Ravindra, Gooldy, Scoville, Guan. Analysis and interpretation of data: Park, Ravindra, de Havenon. Drafting the article: Ravindra. Critically revising the article: all authors. Reviewed submitted version of manuscript: Park, Ravindra, de Havenon. Approved the final version of the manuscript on behalf of all authors: Park. Study supervision: Park, Couldwell.

\section{Correspondence}

Min S. Park, Department of Neurosurgery, Clinical Neurosciences Center, University of Utah, $175 \mathrm{~N}$ Medical Dr. East, Salt Lake City, UT 84132.email: neuropub@hsc.utah.edu. 